\title{
The core/envelope asymmetry in p-mode pulsating stars
}

\author{
A. Miglio ${ }^{1}, 2$, E. Antonello ${ }^{1}$ \\ 1 INAF, Osservatorio Astronomico di Brera, via E. Bianchi 46, 23807 Merate, Italy \\ 2 Institut d'Astrophysique et de Géophysique de I'Université de Liège, Allèe du 6 Août, 17 B-4000 Liège, \\ Belgium
}

\begin{abstract}
It has been shown that there is a potential ambiguity in the asteroseismic determination of the location of internal structures in a pulsating star (Montgomery et al. 2003). We show how, in the case of high-order non-radial acoustic modes, we can possibly remove this ambiguity by considering modes of different degree. To support our conclusions we have investigated the seismic signatures of sharp density variations in the structure of quasi-homogeneous models.
\end{abstract}

\section{Aliasing}

It is known that a sharp variation in the equilibrium structure of a star gives rise to a periodic component in the frequencies of oscillation (see for example Monteiro et al. 2000). A way to isolate these components, in high order modes, is to consider deviations from asymptotic expressions for period (frequency) spacings in g-mode ( $\mathrm{p}$-mode) pulsators.

Montgomery et al. (2003) reported that in the case of white dwarfs, where only high order gravity modes have been detected, there is a potential ambiguity in determining where in the stellar interior the variation that generates the periodic signal is located. With the aim of extending the analysis to acoustic modes, we present how we could possibly remove such an ambiguity by considering modes of different degree.

A general form for the periodic signal generated by a sharp variation located at an acoustic depth $\tau_{d}$ could be approximated by

$$
\delta \nu \simeq A(\nu) \sin \left(2 \pi \nu 2 \tau_{d}+\phi\right)
$$

where $A(\nu)$ is a slowly decreasing function of frequency which depends on the characteristics of the sharp variation (Monteiro et al., 2000). When looking for such a periodic signal in the frequencies of an acoustic oscillation spectrum it is clear that the signal can be evaluated only in a discrete set of frequencies $\nu_{\mathrm{n}, \ell}$, solutions of the oscillation equations.

Let us consider modes of some degree $\ell$ and a periodic signal as in Eq. (1). Having defined the acoustic radius as

$$
\theta(r)=\int_{0}^{r} \frac{\mathrm{dr}^{\prime}}{c}
$$

and remembering the simple first order asymptotic relation (Tassoul, 1980)

$$
\nu_{n} \simeq \Delta \nu\left(n+\phi^{\prime}\right)
$$

and

$$
T \equiv \tau(0) \equiv \theta(R) \simeq 1 /(2 \Delta \nu)
$$


it is straightforward to show that $\delta \nu$ (Eq. (1)) can be also written as

$$
\delta \nu=A(\nu) \sin \left(2 \pi \nu 2 \theta_{d}+\phi^{\prime \prime}\right)
$$

This means that we cannot distinguish whether a variation is located at an acoustic depth $\tau_{d}$ or $\theta_{d}=T-\tau_{d}$ (see Mazumdar \& Antia (2001) and Montgomery et al. (2003) in the case of $\mathrm{g}$ modes).
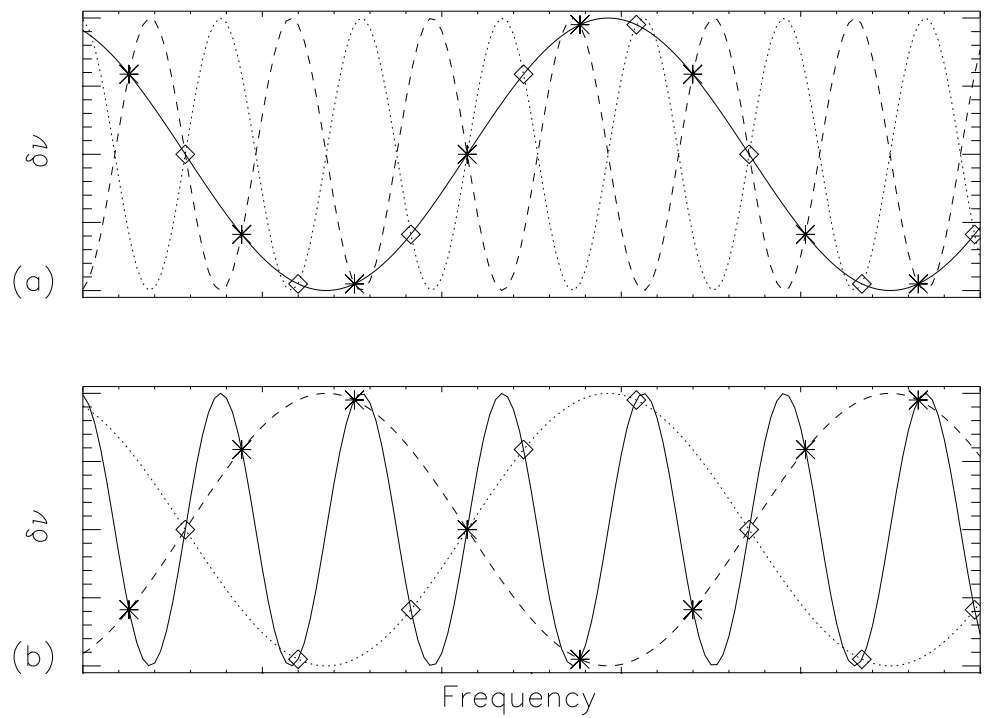

Figure 1: (a) If the discontinuity is located near the surface, the periodic signal can be described as slowly changing and independent from $\ell$ with a period $\sim 1 /\left(2 \tau_{d}\right)$ (continuous line) or as a signal with a short period $\left(1 /\left(2 \theta_{d}\right)\right)$ with a phase which depends on $\ell$ being even or odd (dashed and dotted lines). The signal evaluated at the discrete frequencies in Eq. (5) is represented by asterisks $(\ell=0)$ and diamonds $(\ell=1)$. The values on the axes are arbitrary. (b) The discontinuity is located near the center of the star.

Since we would like to include in our treatment modes of different degree $\ell$ we generalized the previous argument considering, instead of Eq. (2), an asymptotic expression which includes the dependence on $\ell$ (Tassoul, 1980):

$$
\nu_{n, l} \simeq \Delta \nu\left(n+\frac{\ell}{2}+\phi^{\prime}\right)
$$

It follows therefore that the signal in Eq. (1) is equivalent to

$$
\delta \nu=(-1)^{\ell} A(\nu) \sin \left(2 \pi \nu 2 \theta_{d}+\phi^{\prime \prime}\right)
$$

We can then conclude that, to this very first degree of approximation, it is equivalent to consider a periodic signal independent from $\ell$ with a "frequency" twice the acoustic depth or signals that have a "frequency" twice the acoustic radius of the discontinuity and depend on $\ell$ through the multiplicative factor $(-1)^{\ell}$. This can be applied both to periodic signals generated by discontinuities located near the surface and the center of the star (see Fig. 1). 


\section{Signatures of density variations in quasi-homogeneous models}

As a first investigation we considered models with simple density profiles, then found analytically the pressure profile and calculated acoustic oscillation spectra using the Aarhus Adiabatic Pulsation Package ${ }^{1}$. We looked for oscillatory signals in $D_{n \ell}=\left(\nu_{n, \ell}-\nu_{n-1, \ell+2}\right) /(4 \ell+6)$.

As presented in Fig. 2, if we consider a homogeneous model, $D_{n \ell}$ does not depend on the degree $\ell$ and has a smooth behaviour throughout the range of frequencies considered. If we introduce a density variation in the equilibrium model (a step function in the derivative of the density profile) we notice the appearance in $D_{n \ell}$ of a periodic signal whose phase depends on the degree, as qualitatively predicted by Eq. (6).

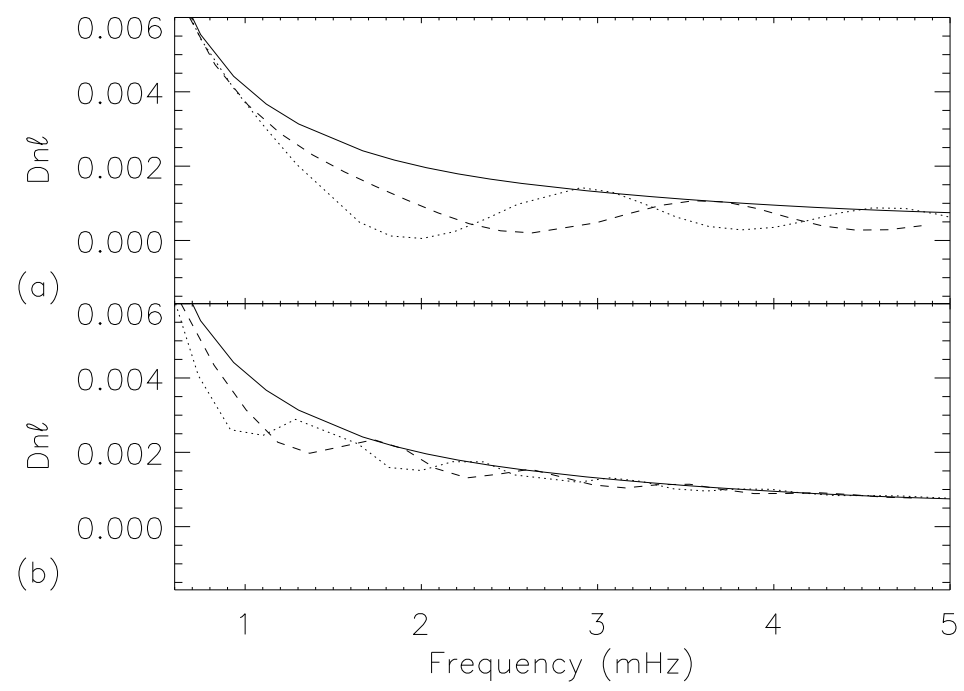

Figure 2: Small separation as a function of frequency. The continuous line is $D_{n 0}\left(=D_{n 1}\right)$ for the homogeneous model; the dashed and dotted lines represent respectively $D_{n 0}$ and $D_{n 1}$ for models with a sharp density variation at (a) $r / R=0.1$ and (b) $r / R=0.3$.

\section{Conclusion}

Using a simple argument we have shown how, in stars that show non-radial acoustic oscillations, we could break the core/envelope symmetry found for g-modes in white dwarfs. It is just a first approximation and has to be thoroughly analyzed and applied to realistic models.

\section{References}

Montgomery M. H., Metcalfe T. S. and Winget, D. E. 2003, MNRAS, 344,657

Monteiro M. J. P. F. G., Christensen-Dalsgaard J. and Thompson M. 2000, MNRAS, 316, 165

Mazumdar A. \& Antia, H. M. 2001, A\&A 377, 192

Tassoul M., 1980, ApJS, 43, 469

\footnotetext{
$1_{\text {http: //astro.phys.au.dk/ jcd/adipack.n/ }}$
} 